Arch. Pharm. (Weinheim) 318, 207-209 (1985)

\title{
Regiospezifische Synthese von 2-Phenyl-pyrano[3,4-b]pyranonen (Oxaflavon-Derivaten) mit Tetrahydro-3-pyranon"
}

\author{
Fritz Eiden* und Klaus Th. Wanner
}

Institut für Pharmazie und Lebensmittelchemie der Universität München, Sophienstraße 10, D.8000 München 2

Eingegangen am 7. November 1983

Tetrahydro-3-pyranon (5) reagiert unter definierten Bedingungen mit Trimethylsilylchlorid regiospezifisch zum Enolether 6. Nach Spaltung mit Methyllithium und Reaktion mit Zimtsäurechlorid entsteht daraus das 4-Cinnamoylpyranon 8. Protonenkatalysierter RingschluB und DDQ-Dehydrierung liefern die Pyrano[3,4-b]pyranone 9 bzw. 10.

\section{Regiospecific Synthesis of 2-Phenylpyrano[3,4-b]pyranones (Oxaflovone Derivatives) with Tetrahydro-3-pyranone}

Under controlled conditions tetrahydro-3-pyrarione (5) reacts with trimethylchlorosilane regiospecifically to yield the enolether 6 . Cleveage with methyllithium and reaction with cinnamoyl chloride furnishes the 4-cinnamoylpyranone 8. Proton catalysed cyclisation and dehydrogenation with DDQ afford the pyrano[3,4-b]pyranones 9 and 10.

In der Natur vorkommende Pyrano[3,4-b]pyrane können bemerkenswerte physiologische Eigenschaften zeigen: Das Rotenon (1) 2.B. ist schon lange als Fisch- und Insektengift bekannt ${ }^{2)}$ und kann als SRS-A-Antagonist wirken'; das Clitoriacetal (2) soll starke antiphlogistische Eigenschaften besitzen".<smiles>C=C(C)C1Cc2c(ccc3c2OC2COc4cc(OC)c(OC)cc4[C@H]2C3=O)O1</smiles><smiles>COc1cc(O)c2c(c1)OC1C(O)Oc3cc(OC)c(OC)cc3[C@@]1(O)[C@H]2O</smiles>

Wir haben die Eignung des Tetrahydro-3-pyranons (5) zur - möglichst regiospezifischen - Synthese von Pyrano[3,4-b]pyranen (3) geprüft. Da bei der Umsetzung von 5 mit Morpholin hauptsächlich das $\Delta^{2}$-Derivat 4 entsteht, das sich zur Darstellung von Pyrano(3,2-b]pyranen eignet ${ }^{1)}$, haben wir die Reaktion von 5 mit Trimethylsilylchlorid (TMS) untersucht und gefunden, daß beim Erhitzen von 5 mit TMS und Triethylamin in THF in sehr guter Ausbeute ausschließlich der $\Delta^{3}$-Enolether (6) entsteht ${ }^{5}$.

$0365-6233 / 8510303-0207 \$ 02.5010$

- VCH Verlagsgeselkschaft mbH, Weinheim 1985 
<smiles>[R12]C1CCCCC1</smiles>

4<smiles>COC1=CC[C@@H](CC(=O)C2CCCOC2)CO1</smiles>

6

Um die bei Reaktionen von Silylenolethern mit Säurechloriden beschriebenen Schwierigkeiten ${ }^{6}$ zu umgehen, haben wir 6 mit Methyllithium versetzt und zur so gebildeten Lösung des Lithiumenolats 7 Zimtsäurechlorid $(\mathrm{ZCl})$ gegeben. Nach dem Aufarbeiten und Umkristallisieren erhielten wir das 4-Cinnamoyl-pyranon 8 in 65proz. Ausbeute.

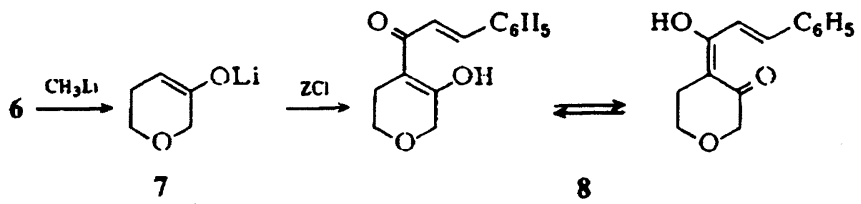

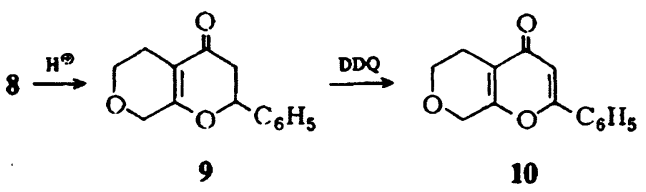

8 ließ sich durch Erhitzen in Ameisensäure zum Pyrano[3,4-b]pyranon 9 cyclisieren, einem Oxaflavanon-Derivat, das sich mit Dichlordicyanochinon (DDQ) unter Bildung von 10 dehydrieren ließ.

Der Ringschlu $B 809$ läßt sich mit Hilfe des ${ }^{1}$ H-NMR-Spektrums von 9 beweisen ( $\delta$-Werte,ppm): das 2H-Signal liegt bei 5.43 (X-Teil eines ABX-Musters), die Signale der beiden Protonen in 3-Stellung liegen bei $2.5-3.3 \mathrm{ppm}$ (AB-Teil, z.T. verdeckt); Lage und Aufspaltung der Signale sind für Flavanon-Derivate typisch" ${ }^{7}$. Außerdem fehlen die im Spektrum von 8 auftretenden Olefin-Signale. Nach der Reaktion von 9 mit DDQ sind im Spektrum von 10 die 2-H- und 3-H-Signale verschwunden. statt dessen ist nur noch das olefinische 3-H-Signal als Singulett bei 6.75 ppm zu erkennen.

Dem Fonds der Chemischen Industrie danken wir für die finanzielle Unterstützung unserer Arbeit.

\section{Experimenteller Teil}

\section{5,6-Dihydro-3-trimeshylsilyloxy-2H-pyran (6)}

$30.0 \mathrm{~g}(300 \mathrm{mmol}) \mathrm{s}^{1)}, 81,5 \mathrm{~g}(750 \mathrm{mmol})$ Trimethylchlorsilan, $83.5 \mathrm{~g}(825 \mathrm{mmol})$ Triethylamin und $200 \mathrm{ml}$ absol. THF wurden $48 \mathrm{~h}$ rückfließend erhitzt. Das Lösungsmittel wurde i.Vak. abgedampft. $300 \mathrm{ml}$ Pentan zugesetzt, filtriert und das Filtrat destilliert. Farblose Flüssigkeit, Sdp. ${ }_{10} 67^{\circ}$, Ausb. $40.9 \mathrm{~g}(79 \%)$. $\mathrm{C}_{8} \mathrm{H}_{16} \mathrm{O}_{2} \mathrm{Si}$ (172.3) Ber. C 55.8 H 9.36 Si 16.3; Gef. 55.9 H 9.30 Si 16.1 Mol.-Masse 172 (ms). - IR(KBr): 2960, 1680,1245 $\mathrm{cm}^{-1}$; $-{ }^{1} \mathrm{H}-\mathrm{NMR}\left(\mathrm{CDCl}_{3}\right): \delta(\mathrm{ppm})=0.20(\mathrm{~s}, 9 \mathrm{H}), 1.92-2.32$ $(\mathrm{m}, 2 \mathrm{H}), 3.67(\mathrm{t}, \mathrm{J}=5.5 \mathrm{~Hz}, 2 \mathrm{H}), 3.78-3.95(\mathrm{~m}, 2 \mathrm{H}), 4.78-5.05(\mathrm{~m}, 1 \mathrm{H})$. 
4-Cinnamoyl-5,6-dihydro-2H-pyran-3(4H)-on (8)

Eine Mischung aus $3.44 \mathrm{~g}(20 \mathrm{mmol}) 6$ und $60 \mathrm{ml}$ absol. THF wurde mit $20 \mathrm{mmol}$ Methyllithiumlösung (1.6 M in Ether) versetzt und 1,5 h bei Raumtemp. gehalten. Die Lösung wurde i. Vak. $\left(10^{-2}\right.$ Torr) bei $0^{\circ}$ auf $1 / 2$ bis $1 / 3$ eingeengt, auf $-78^{\circ}$ abgekühlt und mit $30 \mathrm{ml}$ absol. Toluol verdünnt. Dazu ließ man unter Rühren $1.67 \mathrm{~g}(10 \mathrm{mmol})$ Zimtsäurechlorid in $2 \mathrm{ml} \mathrm{THF}$ tropfen und hielt noch $30 \mathrm{~min}$ bei dieser Temp. Nach Erwärmen auf $-10^{\circ}$ wurde in eine eisgekühlte Mischung aus $100 \mathrm{ml}$ Pentan und $20 \mathrm{ml}$

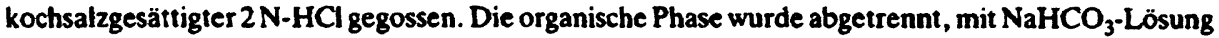
und Wasser geschüttelt und getrocknet (Natriumsulfat). Ein dabei aufgetretener Feststoff wurde isoliert. Die Pentanphase wurde nach Schütteln mit Wasser und nach Trocknen mit Natriumsulfat i.Vak. eingeengt. Durch Verreiben mit Ethylacetat wurde der Rückstand zur Kristallisation gebracht. Die so gewonnenen Kristalle wurden zusammen mit dem aus dem Hydrolysegemisch gewonnenen Feststoff aus Ethylacetat umkristallisiert. Gelbe Kristallplättchen; Schmp. 106 ${ }^{\circ}$, Ausb. $1.5 \mathrm{~g}$ (65\%). $\mathrm{C}_{14} \mathrm{H}_{14} \mathrm{O}_{3}$ (230.3) Ber. C 73.0 H 6.13; Gef. C $72.8 \mathrm{H} 6.06$ Mol.-Masse 230 (ms). - IR(KBr): 1635 , $1575 \mathrm{~cm}^{-1} ;-{ }^{1} \mathrm{H}-\mathrm{NMR}\left(\mathrm{CDCl}_{3}\right): \delta(\mathrm{ppm})=2.67(\mathrm{t}, \mathrm{J}=5.5 \mathrm{~Hz}, 2 \mathrm{H}), 3.87(\mathrm{t}, \mathrm{J}=5.5 \mathrm{~Hz}, 2 \mathrm{H}), 4.18(\mathrm{~s}, 2 \mathrm{H})$, $6.78(\mathrm{~d}, \mathrm{~J}=15.5 \mathrm{~Hz}, 1 \mathrm{H}), \quad 7.22-7.62(\mathrm{~m}, 5 \mathrm{H}), 7.72(\mathrm{~d}, \mathrm{~J}=15.5 \mathrm{~Hz}, 1 \mathrm{H}), 16.00\left(\mathrm{~s}, 1 \mathrm{H}, \mathrm{D}_{2} \mathrm{O}\right.$. Austausch).

\section{2,3,5,6-Tetrahydro-2-phenyl-pyrano[3,4-b]pyran-4(8H)-on (9)}

$4.0 \mathrm{~g}(17.4 \mathrm{mmol}) 8$ wurden in $500 \mathrm{ml}$ Ameisensäure $3 \mathrm{~h}$ rückfließend erhitzt. Die Ameisensäure wurde i.Vak. abgedampft und der Rückstand aus Methanol umkristallisiert. Farblose Kristalle, Schmp. $68-71^{\circ}$, Ausb. $1.65 \mathrm{~g}$ (41\%). Wiederholte Nachkristallisation lieferte weitere $0.9 \mathrm{~g}(22 \%)$. $\mathrm{C}_{14} \mathrm{H}_{14} \mathrm{O}_{3}$ (230.3) Ber. C 73.0 H 6.13; Gef. C $73.0 \mathrm{H} 6.11 \mathrm{Mol}$.-Masse 230 (ms). - IR(KBr): 1665, $1625,1150 \mathrm{~cm}^{-1} .{ }^{1} \mathrm{H}-\mathrm{NMR}\left(\mathrm{CDCl}_{3}\right): \delta(\mathrm{ppm})=2.43(\mathrm{tt}, \mathrm{J} \cdot 6 / 1,5 \mathrm{~Hz}, 2 \mathrm{H}), 2.5-3.3(\mathrm{~m}, 2 \mathrm{H}), 3.42-4.10$ $(\mathrm{m}, 2 \mathrm{H}), 4.18(\mathrm{t}, \mathrm{J}=1.5 \mathrm{~Hz}, 2 \mathrm{H}), 5.43(\mathrm{dd}, 1 \mathrm{H}), 7.43(\mathrm{~s}, 5 \mathrm{H})$.

\section{5,6-Dihydro-2-phenyl-pyrano[3,4-b]pyran-4(8H)-on (10)}

$2.53 \mathrm{~g}$ (11 mmol) 9 und $3.18 \mathrm{~g}$ (14 mmol) 2,3-Dichlor-5,6-dicyan-p-benzochinon wurden in $30 \mathrm{ml}$ absol. Dioxan $12 \mathrm{~h}$ rückfließend erhitzt. Nach Abkühlen und Zusatz von $100 \mathrm{ml}$ Diethylether wurde filtriert und das Filtrat mit $100 \mathrm{ml}$ Ethylacetat verdünnt. Die org. Lösung wurde mit $0.5 \mathrm{~N}-\mathrm{NaOH}$ und mit Wasser geschüttelt, getrocknet (Natriumsulfat) und i.Vak. eingeengt. Aus Methanol blaßgelbe Kristalle, Schmp. 161-163 ${ }^{\circ}$, Ausb. $1.30 \mathrm{~g}(52 \%)$. $\mathrm{C}_{14} \mathrm{H}_{12} \mathrm{O}_{3}$ (228.3) Ber. C 73.7 H 5.30; Gef. C $73.3 \mathrm{H}$ 5.29 Mol.-Masse 228 (ms). - IR(KBr): $1660,1610,1450 \mathrm{~cm}^{-1} ;-{ }^{1} \mathrm{H}-\mathrm{NMR}\left(\mathrm{CDCl}_{3}\right): \delta$ (ppm) = 2.63 $(\mathrm{tt}, \mathrm{J}=5.5 / 1.5 \mathrm{~Hz}, 2 \mathrm{H}), 3.93(\mathrm{t}, \mathrm{J}=5.5 \mathrm{~Hz}, 2 \mathrm{H}), 4.53(\mathrm{t}, \mathrm{J}=1.5 \mathrm{~Hz}, 2 \mathrm{H}), 6.75(\mathrm{~s}, 1 \mathrm{H}), 7.25-7.92$ $(\mathrm{m}, \mathbf{5 H})$.

\section{Literatur}

1 106. Mitt. über Untersuchungen an Pyran-Derivaten; 105. Mitt.: F. Eiden und K.Th. Wanner, Arch. Pharm. (Weinheim) 317, 958 (1984).

2 L. Feinstein und M. Jacobsen, Fortschr.Chem.Org.Naturst. 10, 423 (1953); L. Crombie, ibid. 21 , 275 (1963); E. Wong, ibid. 28, 22 (1970).

3 R.J. Ashack, L.P. McCasty, R.S. Maleek, F.R. Goodman und N.P. Peet, J. Med. Chem. 23, 1022 (1980); N.P. Peet und S. Sunder, J. Heterocycl. Chem. 18, 1123 (1981).

4 T. Tagueki, P. Kanchanapee und T. Amatayakue, Chem. Pharm. Bull. 25, 1026 (1977).

5 J.A. Hirsch und X.L. Wang, Synth. Commun. 12, 333 (1982), konnten 6 nur durch chromatographische Trennung eines Gemisches der $\Delta^{2}$. und $\Delta^{3}$-Isomere erhalten.

6 J.K. Rasmussen, Synthesis 1977, 91 und dort zit. Lit. .

7 T.J.Mabry, K.R. Markham und M.B. Thomas, The Systematic Identification of Flavonoids, S.275, Springer-Verlag, Berlin-Heidelberg-New York 1970. 